Atmos. Chem. Phys., 17, 14811-14819, 2017

https://doi.org/10.5194/acp-17-14811-2017

(C) Author(s) 2017. This work is distributed under

the Creative Commons Attribution 3.0 License.

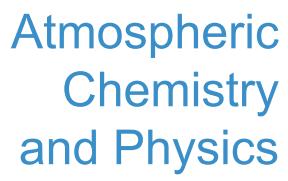

(c) (P)

\title{
Retrieving characteristics of inertia gravity wave parameters with least uncertainties using the hodograph method
}

\author{
Gopa Dutta, Palla Vinay Kumar, and Salauddin Mohammad \\ Vignana Bharathi Institute of Technology, Hyderabad 501301, India \\ Correspondence: Gopa Dutta (gopadutta@yahoo.com)
}

Received: 11 January 2017 - Discussion started: 23 March 2017

Revised: 26 October 2017 - Accepted: 30 October 2017 - Published: 12 December 2017

\begin{abstract}
We have analyzed wind velocities measured with high-resolution Global Positioning System (GPS) radiosondes which have been flown continuously from Hyderabad for $120 \mathrm{~h}$ with an interval of $6 \mathrm{~h}$. Hodograph method has been used to retrieve inertia gravity waves (IGWs) parameters. Background winds are removed from the time series by detrending, whereas polynomials of different orders are removed to obtain the fluctuations from individual profiles. A Butterworth filter is used to extract the monochromatic IGW component. Another filter finite impulse response (FIR1) is tried in a similar manner to test the effects of filters in estimating IGW characteristics. Results reveal that the fluctuation profiles differ with the change in polynomial orders, but the IGW parameters remain same when a Butterworth filter is chosen to extract the monochromatic wave component. The FIR1 filter produces results with a broader range. The direction of wave propagation can be confirmed with additional temperature information.
\end{abstract}

\section{Introduction}

It is well documented that gravity waves of different scales play an important role in maintaining the large-scale circulation of the middle atmosphere. A number of studies have been carried out to characterize these waves by using different techniques. A very common, established and standard procedure of characterizing inertia gravity waves (IGWs) with frequencies close to Coriolis frequency is by the hodograph method (Guest et al., 2000; Ogino et al., 2006; Niranjan Kumar et al., 2011). Radiosonde data of horizontal winds and temperature have been extensively used to study these waves (Tsuda et al., 2004; Vincent and Alexander,
2000; Gong et al., 2008; Chane Ming et al., 2010, 2014; Murphy et al., 2014; Kramer et al., 2015). Nastrom and VanZandt (1982) reported good accuracy in gravity wave parameters derived using balloon measurements since balloons have good aerodynamic responses. In a simulation study Wei and Zhang (2014) have demonstrated that gravity waves with different frequencies and generated by different sources, such as jet imbalance and convection, can coexist. The popular hodograph method demands the presence of a single coherent wave in the fluctuation profiles and does not yield good result when a mixture of various frequencies is present. The gravity wave parameters extracted by the hodograph method might also be inaccurate when multiple waves are present in the data (Eckermann and Hocking, 1989).

The hodograph method is based on a linear theory of gravity waves, whereas the dynamics of the flow is more complex and nonlinear, which introduces some uncertainties in the interpretations of gravity waves. There are several sources of error in this method, which have been described in Zhang et al. (2004). These authors compared the gravity wave characteristics obtained using the hodograph method with the values derived from 4-D output of their simulation study. A narrow bandwidth filter used by them to extract the fluctuations of a near-monochromatic wave resulted in large uncertainties in the horizontal wavelength, which was reduced for waves with shorter vertical wavelengths. Even the spatial variations in the wave characteristics were found to be large. Moreover, since the hodographs are quite variable, a large number of hodographs (profiles) are required to obtain accurate results of gravity wave parameters with some statistical significance (Hall et al., 1995). This limits the very advantage of the hodograph method, which is used to retrieve gravity wave $(\mathrm{GW})$ 

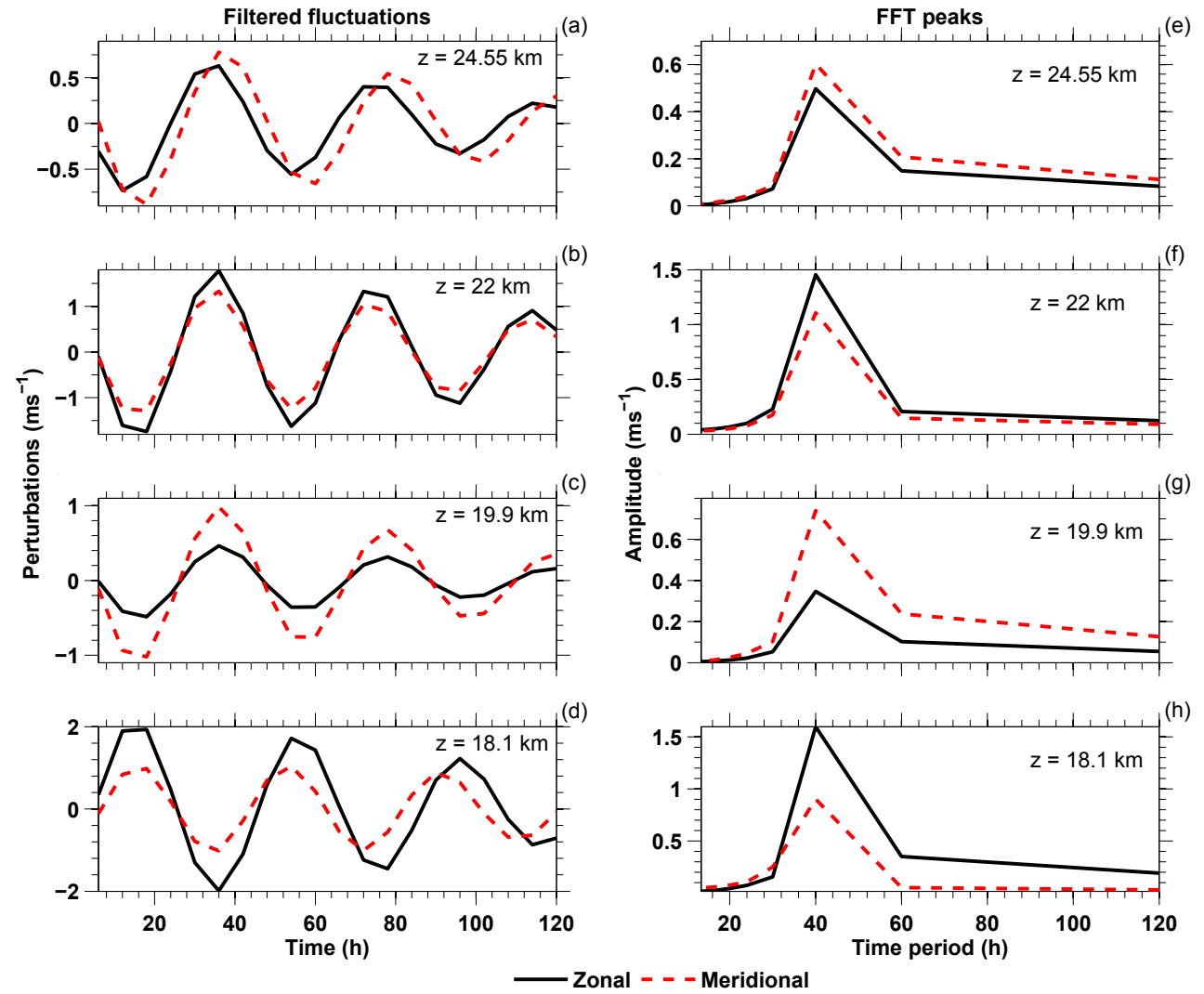

Figure 1. Time series of filtered (Butterworth filter) fluctuations $\left(\mathrm{m} \mathrm{s}^{-1}\right)$ of zonal and meridional winds (a-d) and corresponding FFT spectra (e-f) at a few heights.

parameters from a single set of vertical profiles of zonal and meridional winds.

In this study, we have attempted to reduce uncertainties associated with the hodograph method in delineating the characteristics of IGW from wind velocities obtained with radiosonde measurements.

\section{Experiment and data}

An intensive campaign with high-resolution (i-Met, USA) GPS-radiosonde flights was carried out from the campus of India Meteorological Department (IMD), Hyderabad $\left(17.4^{\circ} \mathrm{N}, 78.5^{\circ} \mathrm{E}\right)$, with four flights a day at an interval of $6 \mathrm{~h}$ for 5 consecutive days (20 flights) between 30 April and 4 May 2012 to study the characteristics of IGW. The timings of the flights were 05:30, 11:30, 17:30 and 23:30 LT. The accuracy of wind and temperature measurements is $\pm 1 \mathrm{~m} \mathrm{~s}^{-1}$ and $\pm 0.2 \mathrm{~K}$, respectively (Vinay Kumar et al., 2016). There was one data gap at 11:30 LT on 4 May 2012, which was linearly interpolated to obtain continuous time series of wind velocities. High-resolution $(\sim 4-10 \mathrm{~m})$ wind data obtained directly from balloon flights were first sorted in ascending order of height since the balloons occasionally drift downwards by a few meters. The wind profiles were then interpo- lated vertically to have a constant height resolution of $50 \mathrm{~m}$. This method is useful to smooth the profiles and to maintain a good height resolution to delineate gravity wave parameters. The profiles were then visually inspected for outliers. Only four outliers could be identified out of 20 profiles; these were removed, and the gaps were filled up by linear interpolation with height.

\section{Analysis and discussion}

\subsection{Time series analysis}

IGW periods in low latitudes are quite large, which makes their observation using a common spectral analysis method difficult. The normal procedure to find the frequency or period of an atmospheric wave is to have a continuous time series data with appropriate data gaps and to subject it to the fast Fourier transform (FFT) technique. The minimum length of data required for FFT analysis is double the period of the wave (Nyquist frequency) to be identified. Keeping this in mind, experiments were conducted as mentioned in Sect. 2 to obtain wind velocities and temperatures continuously for $120 \mathrm{~h}$ with a regular interval of $6 \mathrm{~h}$ since the IGW period over Hyderabad is $\sim 40 \mathrm{~h}$ and the data contain three cycles 

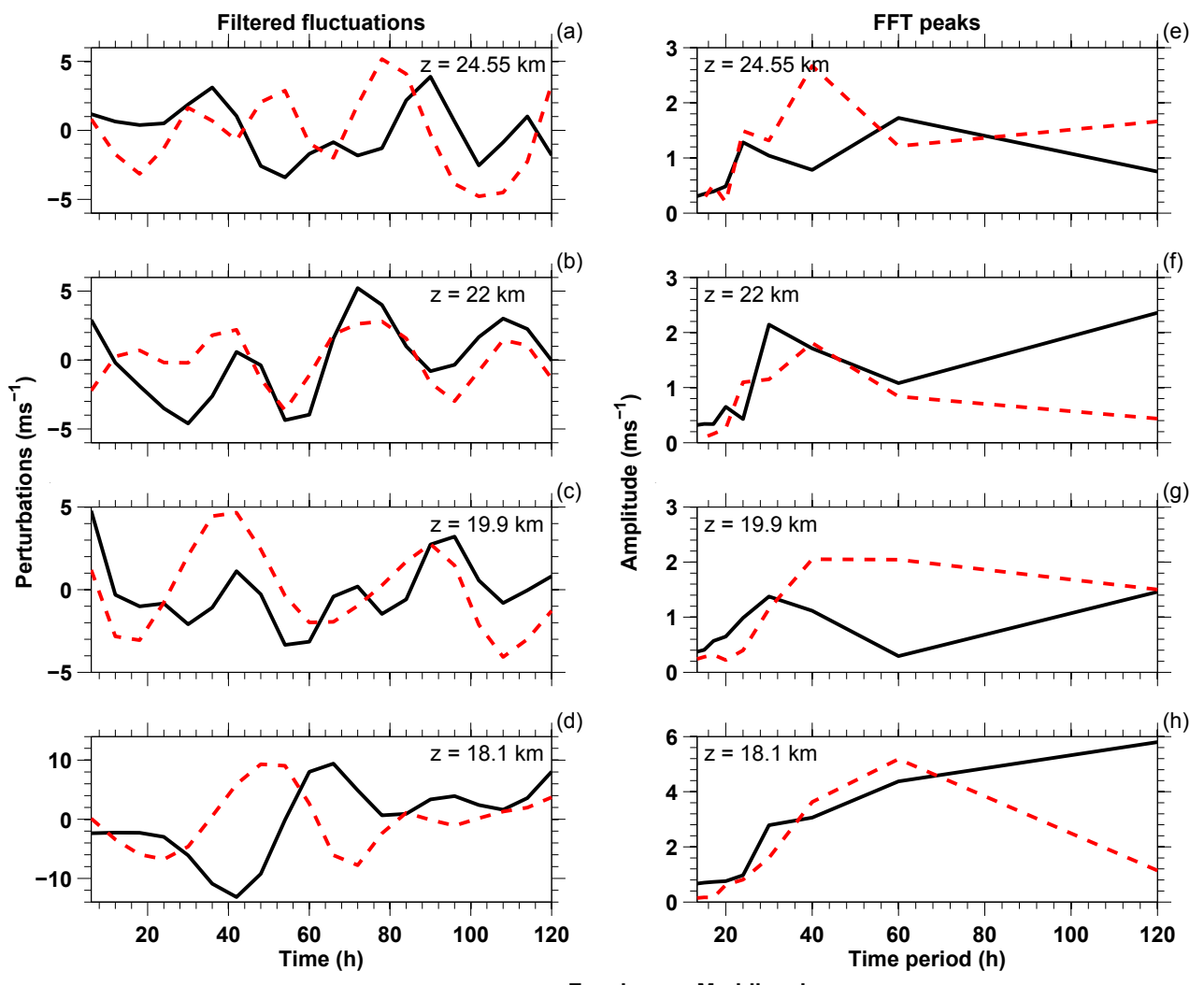

Zonal - - - Meridional

Figure 2. Same as Fig. 1, but with an FIR1 filter.

of the wave, which satisfies the criterion of the FFT technique. These time series data are capable of identifying an IGW period after proper filtering and using a spectral analysis method. These filtered time series data are considered as reference data for the rest of the analyses.

We have used two types of filters: a Butterworth filter and finite impulse response (FIR) filter. A Butterworth filter belongs to the infinite impulse response (IIR) group of filters. It is a type of signal-processing filter designed to have a very flat frequency response in the pass band with a monotonic amplitude response. FIR filters can be reliably designed with a linear phase that prevents distortion. These filters can be easily implemented but with the disadvantage that they often require a much higher filter order than IIR filters to achieve a good level of performance. Further details of these filters are available in Butterworth (1930) and Lake (1980). The order of the filter refers to the number of components that affect the steepness or shape of the filter's frequency response. As the order of the filter increases, the cutoff becomes sharper, but the length of the data should be at least 3 times the filter order. The length of our data is 20 (time-wise), which restricts the maximum order of the filter to be chosen to six. A thirdorder Butterworth filter was found to be more efficient than a sixth-order FIR1 filter for this particular study.

\subsubsection{Hodograph of wind perturbations using a Butterworth filter}

The continuous zonal and meridional wind datasets are detrended (linear trend removed) to obtain time series of wind fluctuations. A third-order Butterworth filter with a bandpass between 36 and $44 \mathrm{~h}$ is applied to the wind perturbations to retrieve the IGW fluctuations with zero phase distortion. The wide band of the time filter is helpful to reduce the Doppler shift of IGW frequency (Niranjan Kumar et al., 2011). Ehard et al. (2015) also recommended the application of a Butterworth filter in extracting gravity waves over a wide range of periods from temperature measured by lidar. The filtered horizontal winds at particular heights are depicted in Fig. 1a-d, which show the presence of IGW with a period of $\sim 40 \mathrm{~h}$. FFT analyses carried out with filtered wind fluctuations also reveal the presence of a clear monochromatic wave of the same period (Fig. 1e-h), which, perhaps, satisfies the requirement of the hodograph method.

Hodographs plotted with these time-wise-filtered zonal and meridional wind perturbations $\left(u_{\mathrm{ew}}^{\prime}, v_{\mathrm{ns}}^{\prime}\right)$ are quite noisy, and it is difficult to identify proper closings. The fluctuation profiles are, therefore, further band-pass filtered using a Butterworth filter with a cutoff at $1.5-4 \mathrm{~km}$, which produced proper elliptic hodographs. The number of proper 

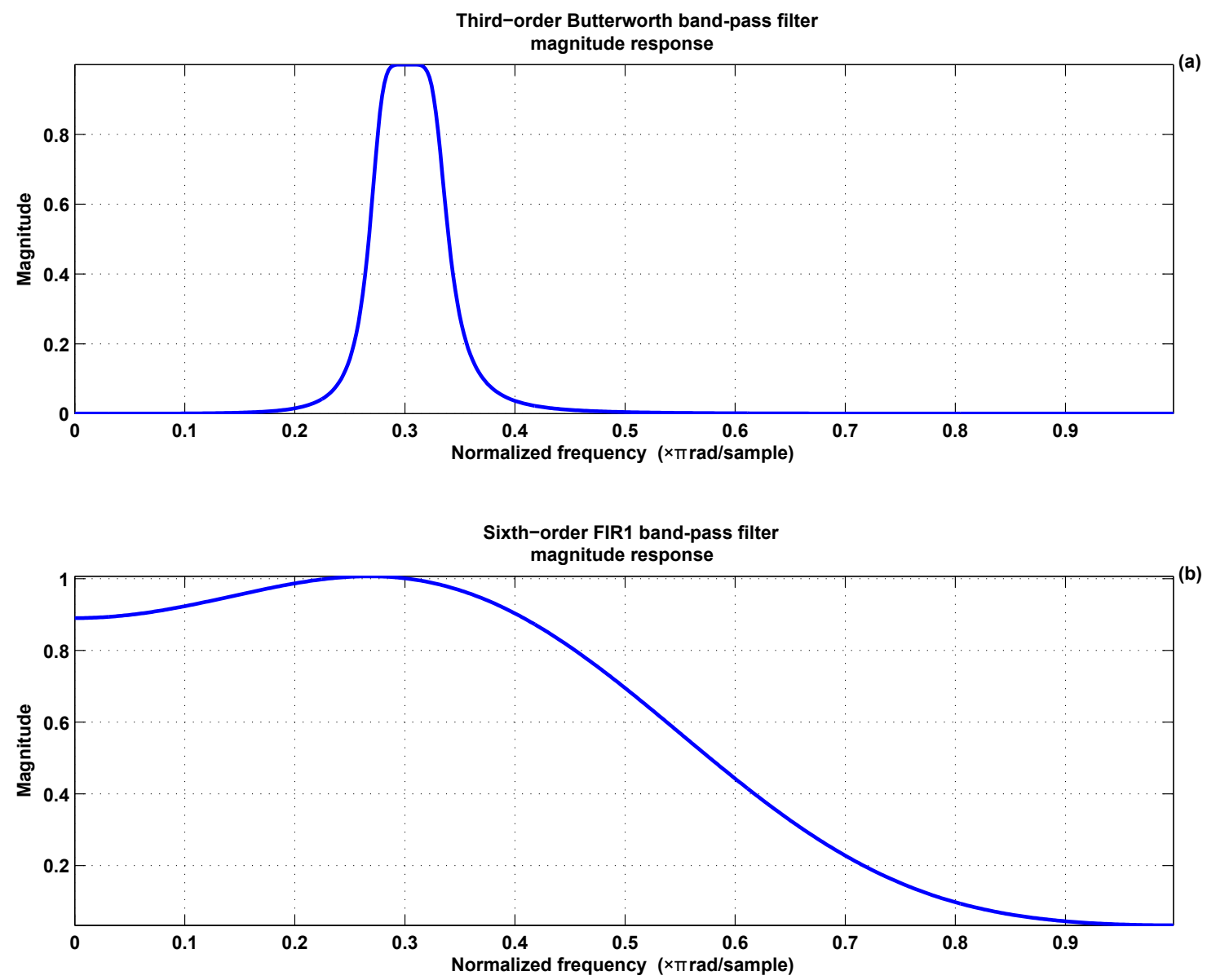

Figure 3. The filter responses of Butterworth (a) and FIR1 (b) filters.

hodographs obtained from 20 pairs of vertical profiles of $u_{\text {ew }}^{\prime}$ and $v_{\mathrm{ns}}^{\prime}$ is 124 . The polarization relation for internal gravity waves is given by Gubenko et al. $(2008,2011)$ :

$\frac{v^{\prime}}{u^{\prime}}=-i\left(\frac{f}{\omega}\right)$

where $u^{\prime}$ and $v^{\prime}$ are the velocity perturbations for the parallel and perpendicular components of wave-induced horizontal wind relative to the wave propagation direction, respectively. This formula implies elliptical wave polarization, with a frequency-dependent ellipse eccentricity of $(f / \omega)$. A few IGW parameters have been extracted using Eq. (1). The horizontal wave number $k$ for internal waves with both low and intermediate intrinsic frequencies $\left(f^{2}<\omega^{2} \ll N^{2}\right)$ is given by the following dispersion equation (Fritts and Alexander, 2003; Gubenko et al., 2012):

$|k|=\left(1-\frac{f^{2}}{\omega^{2}}\right)^{1 / 2} \frac{|m| \omega}{N}$

where parameters $k$ and $m$ represent the horizontal and vertical wave numbers, $N$ is the Brunt-Väisalä frequency, and $f$ and $\omega$ are the inertial (Coriolis parameter) and intrinsic frequencies, respectively. Intrinsic periods of IGW obtained using Eq. (1) from hodographs range between 20 and $28 \mathrm{~h}$, which is less than the inertial period for Hyderabad and belongs to the intermediate range. The vertical and horizontal wavelengths inferred from the hodographs are between 2.0 $2.8 \mathrm{~km}$ and $569-1171 \mathrm{~km}$, respectively.

\subsubsection{Hodographs using an FIR1 filter}

We chose a different sixth-order FIR1 filter to test the effect of filtering on the hodograph method since the vertical wavelength and intrinsic frequency are reported to be highly sensitive to the filter used (Zhang et al., 2004). We followed the same procedure to delineate the IGW parameters as described in Sect. 3.1.1. The detrended and timewise-filtered horizontal wind profiles at a few heights and the corresponding FFT peaks are illustrated in Fig. 2a-d and e$\mathrm{h}$, respectively. Both the time variation in wind fluctuations and the FFT peaks do not show distinct IGW periods. The frequency responses of a third-order Butterworth filter and a sixth-order FIR1 are shown in Fig. 3. The Butterworth filter 
Table 1. Comparison of IGW parameters using detrended time series fluctuations and obtained with different filters.

\begin{tabular}{lrr}
\hline Parameters & Butterworth filter & FIR1 filter \\
\hline Horizontal wavelength $(\mathrm{km})$ & $569-1171$ & $237-1209$ \\
Vertical wavelength $(\mathrm{km})$ & $2.0-2.8$ & $1.5-3.5$ \\
Intrinsic period $(\mathrm{h})$ & $20-28$ & $10-30$ \\
Ratio of minor to major axis & $0.44-0.76$ & $0.35-0.87$ \\
Direction of propagation & southeast (58\%) & southeast (55\%) \\
\hline
\end{tabular}
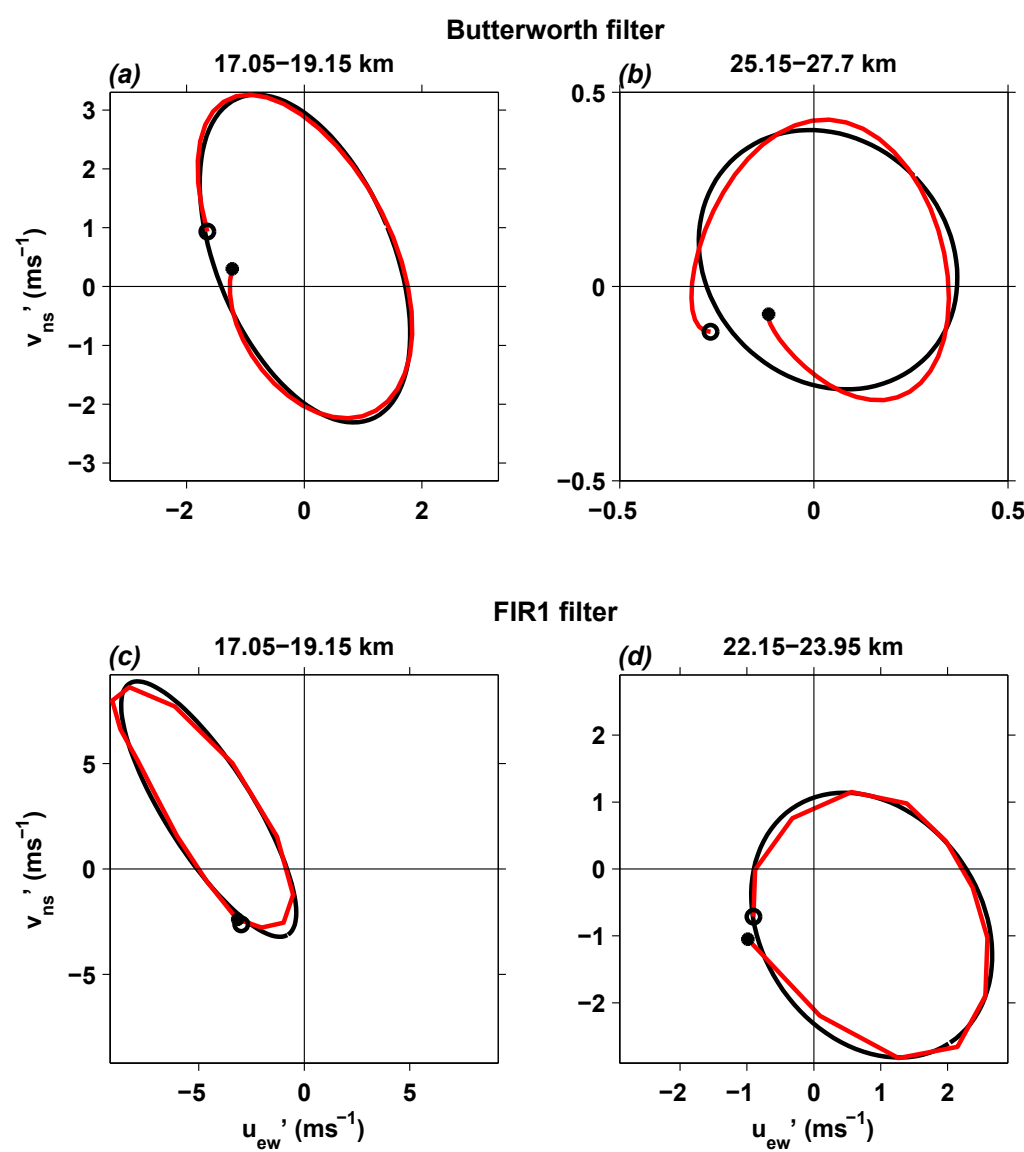

FIR1 filter

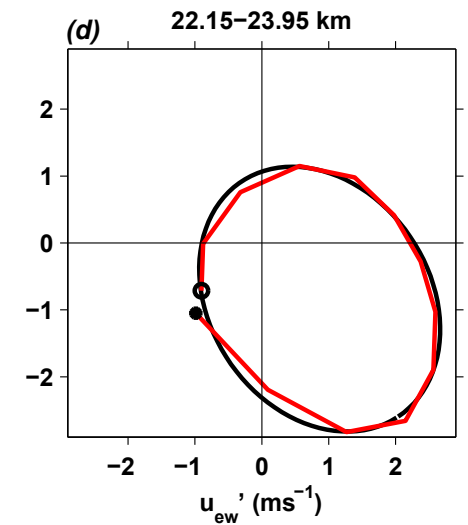

Figure 4. Hodographs of horizontal wind fluctuations $\left(\mathrm{m} \mathrm{s}^{-1}\right)$ obtained using a Butterworth (a, b) and FIR1 (c, d) filters. An open circle and a solid circle in each hodograph indicate the lowest and highest altitudes, respectively. The thin curves represent the elliptical fits.

shows a sharp cutoff and also requires a much lower filter order than the corresponding FIR1 filter. A few hodographs plotted with horizontal wind perturbations using both the filters are displayed in Fig. 4a-d. North is denoted by $0^{\circ}$ in the hodographs, and its orientation angle increases clockwise. Clockwise rotation of the hodograph indicates upward energy propagation in the Northern Hemisphere. The IGW parameters derived from these hodographs are listed in Table 1 . The ranges of horizontal wavelength, vertical wavelength and intrinsic period are broader using an FIR1 filter compared to those obtained using a Butterworth filter.

\subsection{Height series analyses}

Hodographs are generally plotted with the fluctuations derived from data of an individual sounding by removing polynomials of first or second order. We treated the measured vertical profiles of zonal and meridional winds as a single individual set (not time series) and approximated the backgrounds by polynomials of different (two to nine) orders. Figure 5 depicts different fits and the corresponding wind profiles. The fluctuation profiles obtained by removing fourth-, fifth- and sixth-order polynomials show good agreements, whereas appreciable differences could be noticed for others (figure not shown). These fluctuation profiles are then 

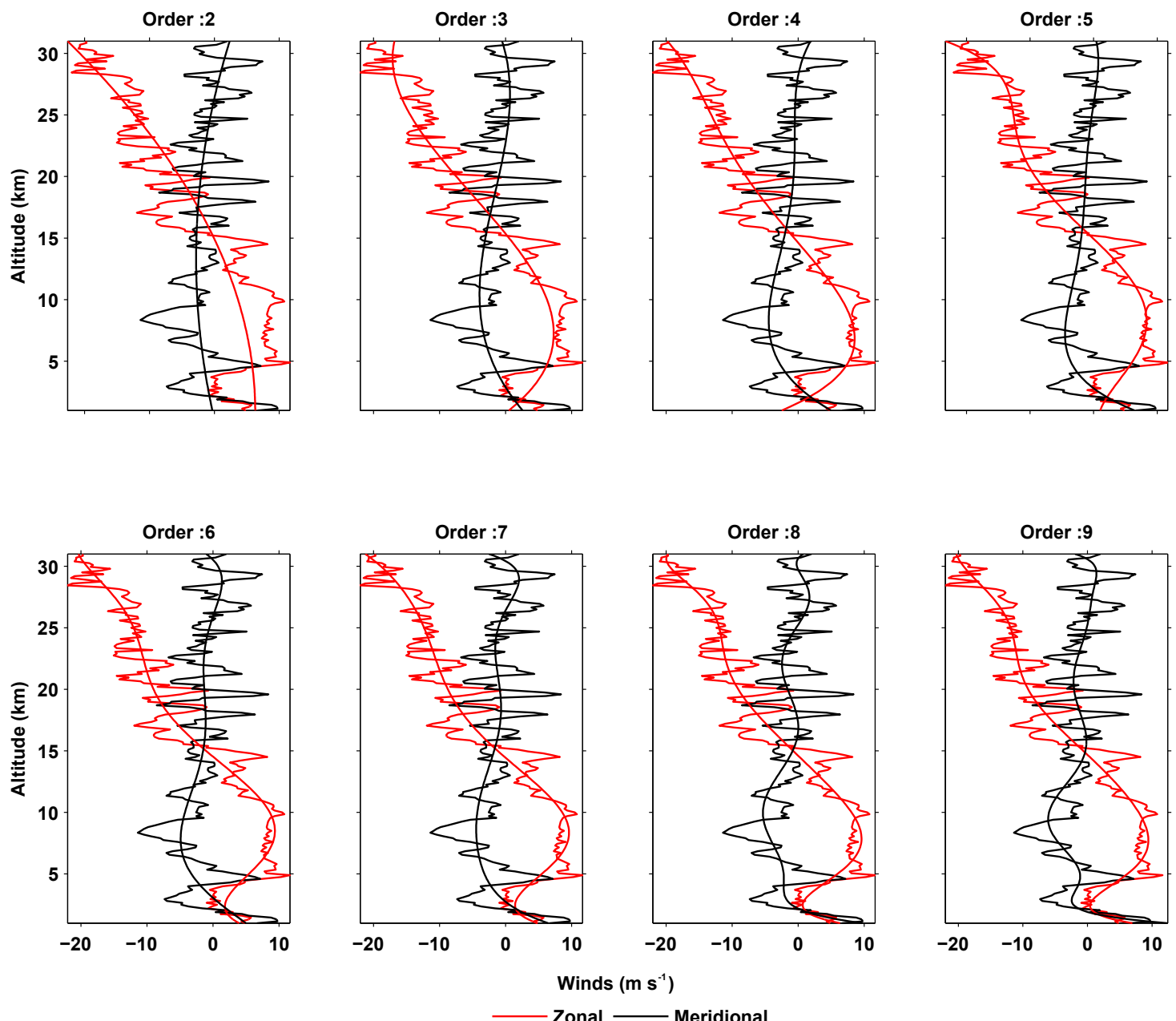

Figure 5. Profiles of zonal and meridional winds $\left(\mathrm{m} \mathrm{s}^{-1}\right)$ and their fits with different orders.

subjected to different filtering process and hodographs are made. They are subsequently analyzed to derive IGW parameters.

\subsubsection{Hodographs using a Butterworth filter}

The perturbation profiles are filtered with a third-order Butterworth filter height-wise to retain IGW oscillations with short vertical wavelengths $(1.5-4 \mathrm{~km})$. IGW parameters obtained from the hodographs plotted with these fluctuations match those described in Sect. 3.1.1 very well.

\subsubsection{Hodographs using an FIR1 filter}

The individual profiles of winds and temperature are then analyzed in a similar manner as mentioned in Sect. 3.2.1 but by using an FIR1 filter with height. The perturbation profiles (after removing backgrounds with different order polynomials) and the filtered fluctuation profiles using both Butterworth and FIR1 filters are shown in Fig. 6a-c and d-f for both the wind components. It can be seen that the Butterworth filter can extract the monochromatic IGW fluctuations very efficiently. The retrieved IGW parameters retain same numerical values (except after decimal points) irrespective of the background removals. Results obtained with an FIR1 filter also belong to the same range but with a broader band, which is illustrated in Table 2 for different orders.

\subsection{Direction of wave propagation}

The direction of horizontal wave propagation is parallel to the major axis of the $u_{\mathrm{ew}}^{\prime}-v_{\mathrm{ns}}^{\prime}$ hodograph (ellipse), which is uncertain by $180^{\circ}$. This uncertainty can be minimized with the help of additional temperature information. Temperature perturbation profiles are obtained by removing fifth-order polynomial fits from the simultaneous temperature profiles and filtering them height-wise with a band-pass Butterworth filter between 1.5 and $4 \mathrm{~km}$. In-phase wind is calculated as $U \cos \theta$, where $U$ is the total wind and $\theta$ is the corresponding orientation angle of the $u_{\mathrm{ew}}^{\prime}-v_{\mathrm{ns}}^{\prime}$ hodograph (Fig. 4a-d). A few hodographs plotted with in-phase winds and temper- 
Table 2. Comparison of IGW parameters using an individual set of wind fluctuation profiles by removing the backgrounds with different order polynomial fits and using both the filters.

\begin{tabular}{|c|c|c|c|c|c|c|}
\hline Filter & $\begin{array}{l}\text { neters } \\
\text { Order number }\end{array}$ & $\begin{array}{r}\text { Horizontal } \\
\text { wavelength }(\mathrm{km})\end{array}$ & $\begin{array}{r}\text { Vertical } \\
\text { wavelength }(\mathrm{km})\end{array}$ & $\begin{array}{r}\text { Intrinsic } \\
\text { period }(\mathrm{h})\end{array}$ & $\begin{array}{l}\text { Ratio of minor } \\
\text { to major axis }\end{array}$ & $\begin{array}{l}\text { Direction of } \\
\text { propagation }\end{array}$ \\
\hline Butterworth & 2 to 9 & $423-986$ & $2.0-2.6$ & $16.0-25.0$ & $0.34-0.71$ & southeast ( $52 \%)$ \\
\hline \multirow[t]{8}{*}{ FIR1 } & 2 & $324-882$ & $1.7-4.0$ & $15.0-23.0$ & $0.34-0.71$ & southeast ( $51 \%)$ \\
\hline & 3 & $472-827$ & $1.7-4.0$ & $17.3-23.9$ & $0.32-0.71$ & southeast $(58 \%)$ \\
\hline & 4 & $404-844$ & $1.7-3.2$ & $15.8-23.5$ & $0.32-0.71$ & southeast $(60 \%)$ \\
\hline & 5 & 273-1090 & $1.8-3.1$ & $16.0-25.0$ & $0.32-0.70$ & southeast $(64 \%)$ \\
\hline & 6 & $361-905$ & $1.7-4.0$ & $15.8-24.7$ & $0.30-0.69$ & southeast $(61 \%)$ \\
\hline & 7 & $440-920$ & $1.7-4.0$ & $16.1-25.4$ & $0.30-0.69$ & southeast $(56 \%)$ \\
\hline & 8 & $360-878$ & $1.8-3.1$ & $16.0-25.0$ & $0.32-0.68$ & southeast ( $55 \%)$ \\
\hline & 9 & $352-739$ & $1.7-4.0$ & $16.2-25.0$ & $0.31-0.68$ & southeast ( $51 \%)$ \\
\hline
\end{tabular}
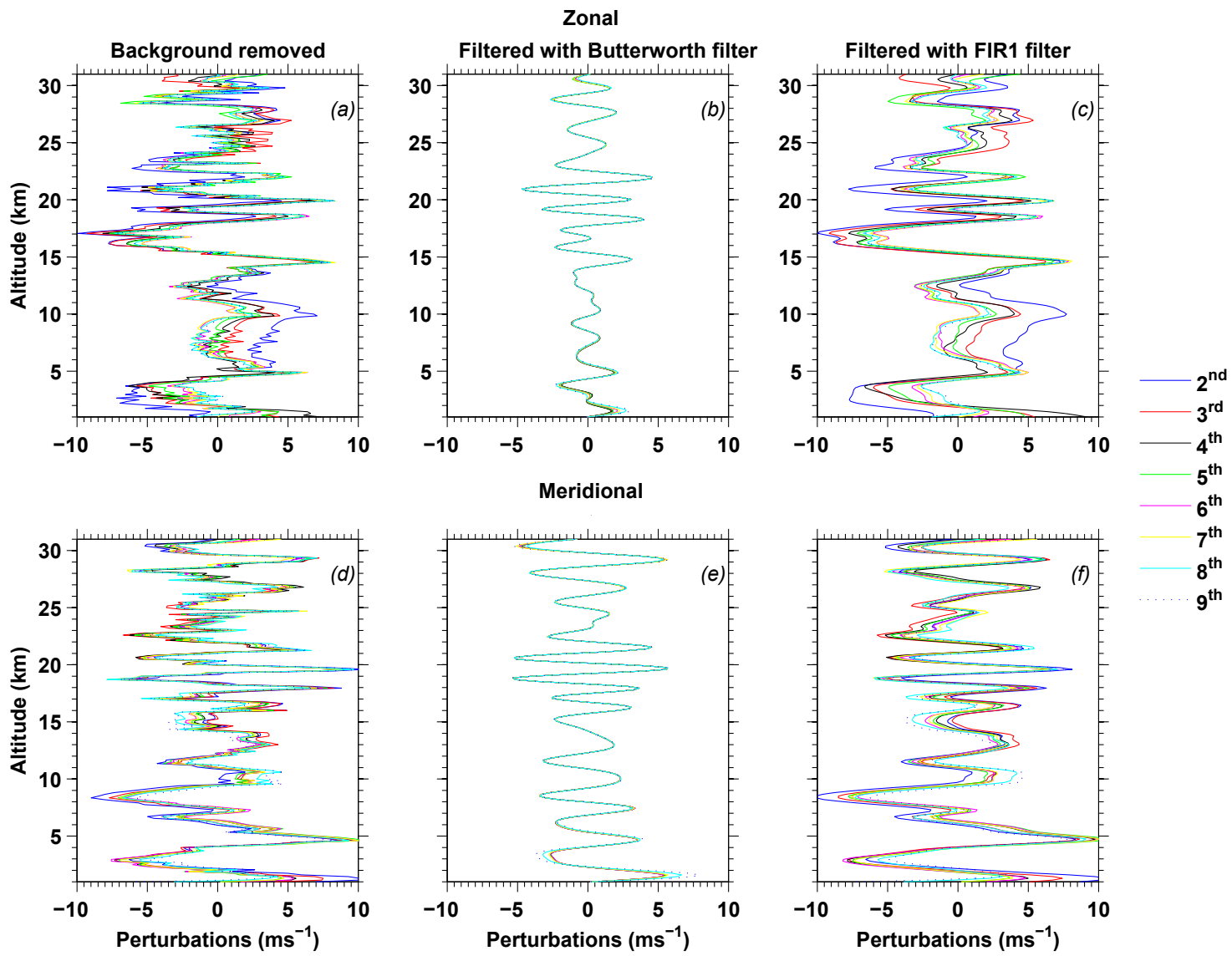

Figure 6. (a, b, c) Vertical profiles of zonal wind fluctuations $\left(\mathrm{m} \mathrm{s}^{-1}\right)$ after approximating the backgrounds with different order (secondninth) polynomials (a) and filtering height-wise with a Butterworth filter (b) and FIR1 filter (c). (d, e, f) Same as upper panel but for meridional wind fluctuations.

ature fluctuations are illustrated in Fig. 7a-d; these help in resolving the ambiguity of wave propagation direction $(\mathrm{Hu}$ et al., 2002). If the rotation of in-phase wind and temperature perturbation hodograph is clockwise, the direction (angle) of horizontal wave propagation will be the same as the orientation angle determined by the $u_{\mathrm{ew}}^{\prime}-v_{\mathrm{ns}}^{\prime}$ hodograph. If the rotation is counterclockwise, it indicates that the propagation direction will be opposite to the orientation angle, i.e., the orientation angle $+180^{\circ}$. As an example, let us consider the hodograph depicted in Fig. 4a. The orientation angle of the major axis of the ellipse is $154.4^{\circ}$. The propagation direction can, therefore, be 154.4 or $154.4+180^{\circ}$. The corre- 

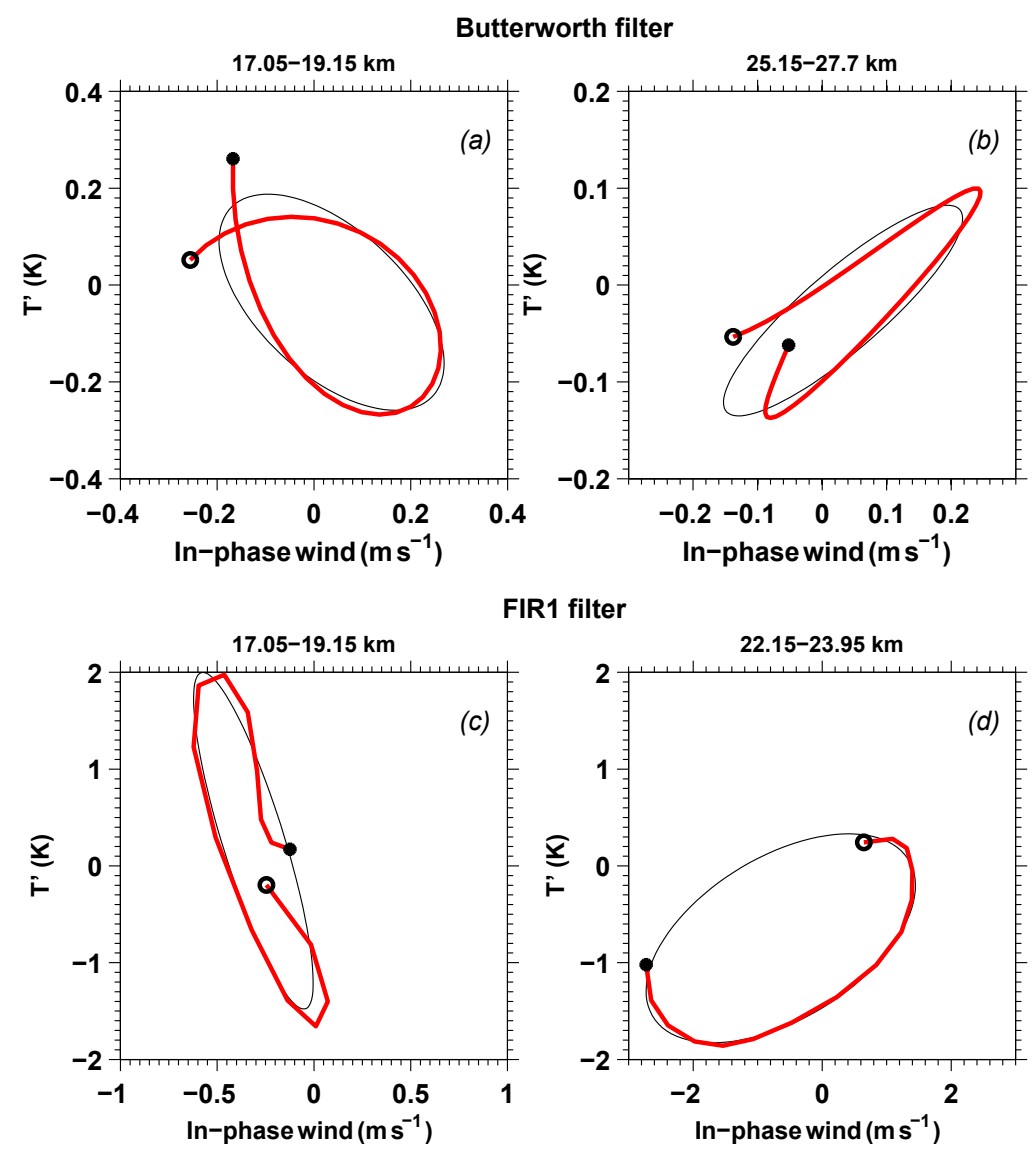

Figure 7. Hodographs of in-phase wind $\left(\mathrm{m} \mathrm{s}^{-1}\right)$ versus temperature fluctuations (K) obtained using Butterworth (a, b) and FIR1 (c, d) filters. An open circle and a solid circle in each hodograph indicate the lowest and highest altitudes, respectively. The thin curves represent the elliptical fits.

sponding in-phase wind and temperature fluctuation hodograph (Fig. 7a) rotates clockwise confirming the propagation direction to be southeast $\left(154.4^{\circ}\right)$. The unambiguous direction of propagation of IGW is observed to be southeast $(58 \%)$ in this study. It is necessary to analyze a large number of hodographs to finalize the direction of propagation.

\section{Summary}

Balloon-borne experiments have been conducted for 5 days with an interval of $6 \mathrm{~h}$ to characterize IGW using the hodograph method. The method is helpful in identifying lowfrequency IGW but suffers from several uncertainties. We have utilized the time series of wind fluctuations to extract the IGW component by filtering and confirmed it with spectral analysis. Results obtained by using Butterworth and FIR1 filters are compared. A band-pass Butterworth filter with a sharp cutoff is found to isolate the monochromatic IGW component very efficiently. Backgrounds of individual wind profiles have been approximated with polynomials of different orders when the perturbation profiles show reason- able differences. The differences are observed to become reduced when a Butterworth filter is used to isolate the IGW components, whereas differences still persist with an FIR1 filter. The IGW parameters delineated from the corresponding hodographs using the former filter agree extremely well for different order polynomial removals. Results obtained with an FIR1 filter also show reasonable agreement but with a broader range. Filtering appears to be of great importance in removing the uncertainties of the hodograph method. The unambiguous direction of wave propagation can be ascertained using additional and simultaneous temperature information.

Data availability. The radiosonde data used in this study are not publicly accessible. However, the data can be provided upon request to Gopa Dutta (gopadutta@yahoo.com). The profiles of winds and temperature are plotted in the Supplement.

The Supplement related to this article is available online at https://doi.org/10.5194/acp-17-14811-2017supplement. 
Competing interests. The authors declare that they have no conflict of interest.

Acknowledgements. The authors are grateful to the Indian Space Research Organization (ISRO), Government of India, for providing financial assistance to run the project under its Climate And Weather of the Sun-Earth System (CAWSES-II) program. The authors wish to thank the India Meteorological Department (IMD), Hyderabad, for their active support in conducting the balloon experiments from their campus. The authors also thank the college management for kind encouragement. Data are available at Vignana Bharathi Institute of Technology, Hyderabad, India. We would like to thank K. Kishore Kumar, Space Physics Laboratory (SPL), for his valuable discussions. We are grateful to the reviewers for their constructive comments, which helped to improve the paper.

Edited by: Jayanarayanan Kuttippurath

Reviewed by: Vladimir Gubenko and one anonymous referee

\section{References}

Butterworth, S.: On the theory of filter amplifiers, Experimental Wireless and the Wireless Engineer, 7, 536-541, 1930.

Chane Ming, F., Chen, Z., and Roux, F.: Analysis of gravity-waves produced by intense tropical cyclones, Ann. Geophys., 28, 531547, https://doi.org/10.5194/angeo-28-531-2010, 2010.

Chane Ming, F., Ibrahim, C., Barthe, C., Jolivet, S., Keckhut, P., Liou, Y.-A., and Kuleshov, Y.: Observation and a numerical study of gravity waves during tropical cyclone Ivan (2008), Atmos. Chem. Phys., 14, 641-658, https://doi.org/10.5194/acp-14-6412014, 2014.

Eckermann, S. D. and Hocking, W. K.: Effect of superposition on measurements of atmospheric gravity waves: a cautionary note and some reinterpretations, J. Geophys. Res., 94, 6333-6339, 1989.

Ehard, B., Kaifler, B., Kaifler, N., and Rapp, M.: Evaluation of methods for gravity wave extraction from middle-atmospheric lidar temperature measurements, Atmos. Meas. Tech., 8, 46454655, https://doi.org/10.5194/amt-8-4645-2015, 2015.

Fritts, D. C. and Alexander, M. J.: Gravity wave dynamics and effects in the middle atmosphere, Rev. Geophys., 41, 1003, https://doi.org/10.1029/2001RG000106, 2003.

Gong, J., Geller, M. A., and Wang, L.: Source spectra information derived from U.S high-resolution radiosonde data, J. Geophys. Res., 113, D10106, https://doi.org/10.1029/2007JD009252, 2008.

Gubenko, V. N., Pavelyev, A. G., and Andreev, V. E.: Determination of the intrinsic frequency and other wave parameters from a single vertical temperature or density profile measurement, J. Geophys. Res., 113, D08109, https://doi.org/10.1029/2007JD008920, 2008.

Gubenko, V. N., Pavelyev, A. G., Salimzyanov, R. R., and Pavelyev, A. A.: Reconstruction of internal gravity wave parameters from radio occultation retrievals of vertical temperature profiles in the Earth's atmosphere, Atmos. Meas. Tech., 4, 2153-2162, https://doi.org/10.5194/amt-4-2153-2011, 2011.
Gubenko, V. N., Pavelyev, A. G., Salimzyanov, R. R., and Andreev, V. E.: A method for determination of internal gravity wave parameters from a vertical temperature or density profile measurement in the Earth's atmosphere, Cosmic Res., 50, 21-31, 2012.

Guest, F., Reeder, M. J., Marks, C. J., and Karoly, D. J.: InertiaGravity wave observed in the lower stratosphere over Macquarie Island, J. Atmos. Sci., 57, 737-752, 2000.

Hall, G. E., Meek, C. E., and Manson, A. H.: Hodograph analysis of mesopause region winds observed by three MF radars in the Canadian prairies, J. Geophys. Res., 100, 7411-7421, 1995.

Hu, X., Liu, A. Z., Gardner, C. S., and Swenson, G. R.: Characteristics of quasi-monochromatic gravity waves observed with $\mathrm{Na}$ lidar in the mesopause region at Starfire Optical Range, NM, Geophys. Res. Lett., 29, 2169, https://doi.org/10.1029/2002GL014975, 2002.

Kramer, R., Wüst, S., Schmidt, C., and Bittner, M.: Gravity wave characteristics in the middle atmosphere during the CESAR campaign at palma de Mallorca in 2011/2012: Impact of extratropical cyclones and cold fronts, J. Atmos. Sol.-Terr. Phy., 128, 8-23, https://doi.org/10.1016/j.jastp.2015.03.001, 2015.

Lake, R.: Programmes for digital signal processing, IEEE Xplore, 50, 31-32, https://doi.org/10.1109/MSP.1980.237616, 1980.

Murphy, D. J., Alexander, S. P., Klekociuk, A. R., Love, P. T., and Vincent, R. A.: Radiosonde observations of gravity waves in the lower stratosphere over Davis, Antarctica, J. Geophys. Res., 119, 11973-11996, https://doi.org/10.1002/2014JD022448, 2014.

Nastrom, G. D. and VanZandt, T. E.: An analytical study of nonlinear responses of rising balloons in horizontal winds, J. Appl. Meteorol., 21, 413-419, 1982.

Niranjan Kumar, K., Ramkumar, T. K., and Krishnaiah, M.: MST radar observation of inertial-gravity waves generated from tropical cyclones, J. Atmos. Sol.-Terr. Phy., 73, 1890-1906, https://doi.org/10.1016/j.jastp.2011.04.026, 2011.

Ogino, S. Y., Sato, K., Yamanaka, M. D., and Watanabe, A.: Lower stratospheric and upper-tropospheric disturbances observed by radiosondes over Thailand during January, 2000, J. Atmos. Sci., 63, 3437-3447, 2006.

Tsuda, T., Ratnam, M. V., May, P. T., Alexander, M. J., Vincent, R. A., and MacKinnon, A.: Characteristics of gravity waves with short vertical wavelengths observed with radiosonde and GPS occultation during DAWEX (Darwin Area Wave Experiment), J. Geophys. Res., 109, D20S03, https://doi.org/10.1029/2004JD004946, 2004.

Vincent, R. A. and Alexander, M. J.: Gravity wave in the tropical lower stratosphere: An observational study of seasonal and interannual variability, J. Geophys. Res., 105, 17971-17982, 2000.

Vinay Kumar, P., Dutta, G., Ratnam, M. V., Krishna, E., Bapiraju, B., Rao, B. V., and Mohammad, S.: Impact of cyclone Nilam on tropical lower atmospheric dynamics, Adv. Atmos. Sci., 33, 955-968, https://doi.org/10.1007/s00376-016-5285-x, 2016.

Wei, J. and Zhang, F.: Mesoscale gravity waves in moist baroclinic jet-front systems, J. Atmos. Sci., 71, 929-952, https://doi.org/10.1175/JAS-D-13-0171.1, 2014.

Zhang, F., Wang, S., and Plougonven, R.: Uncertainties in using the hodograph method to retrieve gravity wave characteristics from individual soundings, Geophys. Res. Lett., 31, L11110, https://doi.org/10.1029/2004GL019841, 2004. 